\title{
Protection scheme for transmission lines based on correlation coefficients
}

\author{
R. Abd Allah \\ Buraydah Colleges, Faculty of Engineering, Electrical Power Department, Qassim Region, Kingdom of Saudi Arabia
}

Email address:

Mohandes_Ragab@yahoo.com

To cite this article:

R. Abd Allah. Protection Scheme for Transmission Lines Based on Correlation Coefficients. International Journal of Energy and Power Engineering. Vol. 3, No. 2, 2014, pp. 93-102. doi: 10.11648/j.ijepe.20140302.18

\begin{abstract}
In modern digital power system protection systems, statistical coefficients technique is recently used for fault analysis. A correlation technique is developed for faults detection and discrimination. The proposed technique is able to accurately identify the condition of phase(s) involved in all ten types of shunt faults that may occur in extra high-voltage transmission lines under different fault resistances, inception angle and loading levels. The proposed technique does not need any extra equipment as it depends only on the three line-currents measurements which are mostly available at the relay location. This technique is able to perform the fault detection, type and phase selection in about a half-cycle period. Thus, the proposed technique is well suited for implementation in digital protection schemes. The suggested scheme is applied for a part of $500 \mathrm{Kv}$ Egyptian network. Alternative transient program (ATP) and MATLAB programs are used to implement the proposed technique.
\end{abstract}

Keywords: Power System, Fault Detection, Fault Classification, Correlation Coefficient

\section{Introduction}

In modern power system protection, accurate, fast and reliable fault classification technique is an important operational requirement. On one hand, correct information of the type of fault is readily needed for fault location algorithms [1]. On the other hand, in digital distance protection schemes, for proper operation of the protective relays, correct determination of the fault type is a prerequisite [2]. A significant amount of research work has been directed to address the problem of an accurate fault classification scheme. Among the various techniques reported for fault classification in transmission system, Artificial-neural-network (ANN) approach is the most widely used techniques. Although the neural-networkbased approaches have been quite successful in determining the correct fault type, the main disadvantage of ANN is that it requires a considerable amount of training effort for good performance, especially under a wide variation of operating conditions such as, system loading level, fault resistance, fault inception instance [3-9]. Similarly, Expert system-based approach effectiveness depends largely on the domain knowledge of the experts, which is often quite time-consuming to be obtained [10-12]. Fuzzy and fuzzy neural-network-based approaches also require extensive training of the ANN [13-14]. Recently, fault classification techniques suitable for a fault recorder has been proposed in [15], which can identify all ten types of short-circuit faults. Some statistical coefficients techniques were used for fault analysis such as an alienation technique was developed for faults detection and discrimination. The paper [16] presented protection scheme for transmission lines based on alienation coefficients for current signals; the scheme used another algorithm besides alienation coefficients to distinguish between double phase and double phase-to-ground faults. In this paper, a fault detection and selection based on correlation technique is proposed which is able to determine accurately, during one cycle period of fundamental frequency, all fault types and phase selection. Also this technique takes into consideration the wide variations of operating conditions such a switching, pre-fault power level, fault resistance and fault inception angle.

\section{Fault Classification Strategy}

The fault selection algorithm is based on the autocorrelation technique of two half successive cycles with the same polarity. For transmission lines protection, this 
method needs only three line-current measurements available at the relay location $\left(i_{a}, i_{b}, i_{c}\right)$.

\subsection{Correlation Coefficients Calculation}

The auto-correlation coefficient is estimated as follows for any two dependant variables, $y_{1}(x)$ and $y_{2}(x)$ [17]. The auto-correlation coefficient $(r)$ calculated as follows:

$$
r=\frac{\sum_{x=1}^{N_{s}} y_{1}(x) y_{2}(x)-N_{s} \bar{y}_{1} \bar{y}_{2}}{\sqrt{\left(\sum_{x=1}^{N_{s}} y_{1}^{2}(x)-N_{s}\left(\bar{y}_{1}\right)^{2}\right)\left(\sum_{x=1}^{N_{s}} y_{2}^{2}(x)-N_{s}\left(\bar{y}_{2}\right)^{2}\right)}}
$$

Where, $N_{s}=$ the number of samples per cycle used in the simulation

$r$ : empirical correlation coefficient of $y_{1}(x)$, and $y_{2}(x)$.

$y_{l}(x)$ : is the initial instantaneous value of the current at time $\mathrm{t}_{0}$.

$y_{2}(x)$ : is the instantaneous value of the current at next cycle.

$\bar{y}_{1}, \bar{y}_{2}$ : arithmetic means of $y_{1}(x)$ and $y_{2}(x)$, respectively.

$$
\begin{aligned}
& \bar{y}_{1}=\frac{1}{N_{s}} \sum_{x=1}^{N_{s}} y_{1}(x) \\
& \bar{y}_{2}=\frac{1}{N_{s}} \sum_{x=1}^{N_{s}} y_{2}(x)
\end{aligned}
$$

The strength of linear association between two variables is quantified by the correlation coefficient $(r)$, its value lies between -1 and +1 [17].

\subsection{Fault Detection and Faulty Phase Selection}

To implement our technique, three tasks are starting in parallel: fault detection, fault confirmation, and faulty phase selection as follows:

\subsubsection{Fault Detection (Initiation)}

A transition is detected if: $\Delta I>20 \% I_{n}$, where $I_{n}$ is the line nominal current.

\subsubsection{Faulty Phase Selection}

- Fault confirmation and faulty phase selection are done according to the following sequences.

Three-phase current correlation coefficients values are calculated. If fault is detected, phase current correlation values are sorted in ascending order and compared.

- If fault is detected, phase current correlation values are sorted into ascending order and compared. The possible fault cases are:

(a) If the three-phase correlation coefficients are nearly equal and their values are less than 0.7 , then the fault is three-phase fau

- If $r_{\mathrm{a}} \approx \mathrm{r}_{\mathrm{b}} \approx \mathrm{r}_{\mathrm{c}}<0.7$, the fault is three-phase (a-b-c fault)

(b) If the two-phase correlation coefficients are equal and their values are nearly 1, while the third phase correlation coefficient is less than 0.7 , the fault is singlephase to ground fault.

- If $r_{a}<0.7, r_{b} \approx 1, r_{c} \approx 1$, the fault is single phase- toground fault (a-g fault)

- If $\mathrm{r}_{\mathrm{b}}<0.7, \mathrm{r}_{\mathrm{a}} \approx 1, \mathrm{r}_{\mathrm{c}} \approx 1$, the fault is single phase- toground fault (b-g fault)

- If $\mathrm{r}_{\mathrm{c}}<0.7, \mathrm{r}_{\mathrm{a}} \approx 1, \mathrm{r}_{\mathrm{b}} \approx 1$, the fault is single phase- toground fault (c-g fault)

(c) If the two-phase correlation coefficients are equal and their values are less than 0.7 , while the third phase correlation coefficient is nearly 1 , the fault is double phaseto-ground fault.

- If $r_{a} \approx r_{b}<0.7, r_{c} \approx 1$ the fault is double phase-toground fault (a-b-g fault)

- If $r_{b} \approx r_{c}<0.7, r_{a} \approx 1$ the fault is double phase-toground fault (b-c-g fault)

- If $r_{a} \approx r_{c}<0.7, r_{b} \approx 1$ the fault is double phase-toground fault (a-c-g fault)

(d) If the three-phase correlation coefficients are not equal and their values: one phase is less than 0.3 , second phase is less than 0.7 , while the third phase alienation coefficient is nearly 1 , the fault is phase-to-phase fault.

- If $\mathrm{r}_{\mathrm{a}}<0.7, \mathrm{r}_{\mathrm{b}}<0.7, \mathrm{r}_{\mathrm{c}} \approx 1$ the fault is phase-to-phase fault (a-b fault)

- If $r_{b}<0.7, r_{c}<0.7, r_{a} \approx 1$ the fault is phase-to-phase fault (b-c fault)

- If $r_{a}<0.7, r_{c}<0.7, r_{b} \approx 1$ the fault is phase-to-phase fault (a-c fault)

- To make sure of distinguishing between double phase and double phase-to-ground faults, the cross-correlation between the two phase currents of the faulted phases is calculated.

If the value of cross-correlation is nearly -1 , the fault is double phase fault.

- If $r_{a b} \approx-1$ the fault is phase-to-phase ( $a-b$ fault) otherwise the fault is double phase-to-ground (a-b-g fault).

- If $r_{b c} \approx-1$ the fault is phase-to-phase (b-c fault) otherwise the fault is double phase-to-ground (b-c-g fault).

- If $\mathrm{r}_{\mathrm{ac}} \approx-1$ the fault is phase-to-phase (a-c fault) otherwise the fault is double phase-to-ground (a-c-g fault).

\section{Case Study Power System}

The proposed technique is applied on the power system shown in Fig.1. The system parameters are obtained from one-generation unit in El-KURIEMAT power station that produces 320 MVA [18]. The parameters of the selected system are as follows:

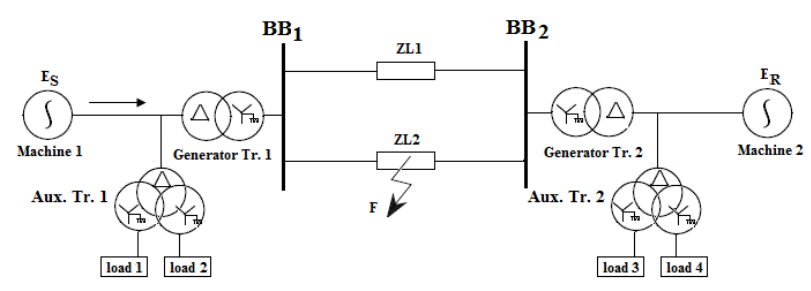

Figure 1. The simulated power system. 


\section{Machine 1 (Sending Source):}

Rated line voltage is $19 \mathrm{kV}$, Volt-Ampere rating is 320 MVA, Frequency is $50 \mathrm{~Hz}$, Voltage phase angle is $20^{\circ}$ and number of poles is 2 .

\section{Machine 2 (Receiving Source):}

Machine 2 has the same parameters of Machine 1 except the steady-state voltage phase angle is $0^{0}$.

\section{Main Transformers:}

At each side there is a step up transformer 340 MVA, $19.57 / 500 \mathrm{kV}$ (Delta/Star earthed neutral), its primary impedance is $0.0027+\mathrm{j} 0.184 \Omega$, its secondary impedance is $0.7708+\mathrm{j} 61.8 \Omega$.

\section{Aux. Transformers:}

At each side there is an auxiliary transformer 32 MVA, 19.57/6.3/6.3 kV (Delta/Star/Star earthed neutral), its primary impedance is $0.02978+\mathrm{j} 0.4894 \Omega$, its secondary impedance is $0.0039+\mathrm{j} 0.0261 \Omega$.

\section{Lines}

Transmission line (T.L.) impedance is $0.0217+\mathrm{j} 0.302 \Omega$ $/ \mathrm{Km}$ with $200 \mathrm{Km}$ length for each circuit.

\section{Loads}

Each load is represented as impedance of value $100.0+$ j60.0 $\Omega$.

\section{Simulation Results}

A fault $(F)$ was considered at the middle of one circuit of the transmission line assuming that short circuit is temporary and not resistive. The developed technique was applied by calculating the auto-correlation coefficient $\left(r_{\mathrm{x}}\right)$ between two successive half cycles with the same polarity of current signals at the sending-end where relays would normally be installed. The proposed technique is capable to discriminate between the two types of two -phase faults either earthed or isolated. To confirm the two faulted phase type, a cross-correlation coefficient is calculated for the faulted phase. To implement the present technique, the studied power system configuration was simulated by using ATP software [19]. The generated and measured three phase line current signals are taken from the transmission line terminals at " $\mathrm{BB}_{1}$ " side. Five simulation case studies are done to discriminate between the faulty phases, by using the proposed method, for fault classification. These cases are done under effects of different pre-fault power level, fault resistance, and fault inception angle located on the simulated power system. The current measured signals sampling rate is 50 samples per cycle, which means sampling time of $0.4 \mathrm{mSec}$. The total simulation time is $2 \mathrm{Sec}$ (i.e. the total number of samples is 5000). The fault inception time is $0.402 \mathrm{Sec}$ and the fault clearing time is $0.502 \mathrm{Sec}$ from the beginning of the simulation time.

\subsection{Three Phase-to-Ground Fault (Case 1)}

This case studies the performance of the proposed technique during the three phase-to-ground fault condition on the proposed technique. The operating power angle of generator $1\left(\delta_{1}\right)$ is 20 Degree. Figure 2 shows the simulation results for case "1". Figures $2(\mathrm{a}-\mathrm{c})$ present the instantaneous values for the three phase currents. In this case, it is noticed that the three phase currents during the fault are higher than the pre-fault currents; their values are nearly five times the pre-fault current. The correlation coefficients $\left(r_{x}\right)$ are calculated between two successive half-cycles with the same polarity for the three-phase current signals. The three-phase current correlation coefficients $r_{i a}, r_{i b}$ and $r_{i c}$ are shown in Figs. 2 (d-f).The values of $r_{i a}, r_{i b}, r_{i c}$ are equal and close to one before fault inception and after fault clearing. At fault start they are equal and less than 0.24 while they are less than 0.40 at fault clearing. From the above results, it is clear that the correlation coefficient values at fault initiation are good detector to determine the faulted phases; Their values are closely to one for current signals in case of normal operation and they are less than one in case of fault condition. Summary of the correlation coefficients for the measured current signals at the different periods are shown in Table 1.

Table 1. Correlation coefficients in case of three phase-to-ground fault $\left(\delta_{l}=20\right.$ degree, three phase-to-ground faults $(a-b-c-g)$ with $\left.R_{f}=0 \mathrm{ohm}\right)$.

\begin{tabular}{lllllll}
\hline Fault type & Signals & $(\mathbf{r})$ pre-fault & $(\mathbf{r})$ at fault start & $(\mathbf{r})$ during fault & (r) at fault clearing & (r) post-fault \\
\hline \multirow{2}{*}{ Three line-to- } & ia & ria $=1$ & 0.24 & 1 & 0.20 & 1 \\
ground fault & ib & rib $=1$ & 0.21 & 1 & 0.35 & 1 \\
& ic & ric $=1$ & 0.23 & 1 & 0.40 & 1 \\
\hline
\end{tabular}

\subsection{Single Line-To-Ground Fault (Case 2)}

All parameters are kept as in case "1", except the fault type is changed from three lines-to-ground fault (a-b-c-g) to single line-to-ground fault (a-g). Figures 3 (a-c) present the instantaneous values for three-phase currents. The faulty phase current value is nearly six times the pre-fault current, while both the healthy phase currents are equal and nearly 1.35 times the pre-fault value. The three phase current correlation coefficients $r_{i a}, r_{i b}, r_{i c}$ are shown in Figs. 3 (d-f). Their values are equal and close to one before fault inception and after fault clearing. At fault start $r_{i a}$ is less than 0.74 while $r_{i b}$ and $r_{i c}$ are nearly one. At fault clearing, $r_{i b}$ is equal one while $r_{i a}, r_{i c}$ are less than 0.74 . From the 
above results, it is clear that the correlation coefficient value at fault initiation is good indicator to determine the faulted phase $A$. Summary of the correlation coefficients for the measured signals at the different periods are shown in Table 2.

Table 2. Correlation coefficients in case of single phase-to-ground fault ( $\delta_{1}=20$ degree, single phase-to-ground faults (a-g) with $R_{f}=0$ ohm).

\begin{tabular}{|c|c|c|c|c|c|c|}
\hline Fault type & Signals & (r) pre-fault & (r) at fault start & (r) during fault & (r) at fault clearing & (r) post-fault \\
\hline \multirow{3}{*}{$\begin{array}{l}\text { Single line-to- } \\
\text { ground fault }\end{array}$} & ia & ria $=1$ & -0.1 & 1 & $0.15-$ & 1 \\
\hline & $\mathrm{ib}$ & $\mathrm{rib}=1$ & 1 & 1 & 1 & 1 \\
\hline & ic & ric $=1$ & 0.74 & 1 & 0.74 & 1 \\
\hline
\end{tabular}

\subsection{Double Line-To-Ground Fault (Case 3)}

This case studies the effect of double line-to-ground fault condition on the performance of the proposed algorithm. Therefore, all parameters are kept as in case "1", except the fault type is changed to double line-to-ground fault (a-c-g). Figures 4(a-f) show the simulation results for three-phase currents as the two faulty phases currents are nearly ten times the pre-fault values while the healthy phase current is nearly 1.35 times its pre-fault current value. The values of correlation coefficients $r_{i a}, r_{i b}$ and $r_{i c}$ are equal and close to one before fault inception and after fault clearing. At fault starts, $r_{i a}$ and $r_{i c}$ are less than the value of 0.71 , whereas $r_{i b}$ is nearly one. At fault clearing, $r_{i a}$ and $r_{i c}$ are less than 0.78 whereas $r_{i b}$ is nearly one. From the above results, it is clear that the correlation coefficient value at fault initiation can define the double line-to-ground fault. The cross-correlation coefficient $r_{i a c}$, calculated between the two faulted phases $(A$ and $C$ ), at fault start has a value of - 0.85 ; this value confirms the type of fault is double line-to-ground fault. Before fault inception, the value of cross-correlation coefficient $r_{i a c}$ is equal to $\cos \left(120^{\circ}\right)=-$ 0.5 , which is considered a normal value. Summary of the correlation coefficients for the measured signals at the different periods is shown in Table 3. From the obtained results, it is clear that the correlation coefficient value at fault initiation is good detector to determine the faulted phases $(A$ and $C)$.

Table 3. Correlation coefficients in case of double phase-to-ground fault $\left(\delta_{l}=20\right.$ degree, double phase-to-ground faults $(b-c-g)$ with $\left.R_{f}=0 \mathrm{ohm}\right)$.

\begin{tabular}{lllllll}
\hline Fault Type & Signals & (r) pre-fault & (r) at fault start & (r) during fault & (r) at fault clearing & (r) post-fault \\
\hline & ia & ria $=1$ & -0.05 & 1 & -0.07 & 1 \\
Double line-to- & ib & rib $=1$ & 0.71 & 1 & 0.78 & 1 \\
ground fault & ic & ric $=1$ & 0.27 & 1 & 0.38 & 1 \\
& ib \& ic & ribc $=-0.5$ & -0.85 & -0.5 & -0.58 & -0.5 \\
\hline
\end{tabular}

\subsection{Double Line Fault (Case 4)}

This case studies the effect of double line fault Condition on the performance of the proposed algorithm. Therefore all parameters are kept as in case "1", except that the fault type is changed to double line fault (a-c). Figures 5(a-f) show the simulation results for the three-phase currents as the two faulty phases currents are nearly ten times the pre-fault values, whereas the healthy phase current is nearly 1.35 its pre-fault value. The values of correlation coefficients $r_{i a}, r_{i b}$ and $r_{i c}$ are equal and close to one before fault inception and after fault clearing. At fault start, $r_{i a}, r_{i b}$ and $r_{i c}$ have different values with values of $0.55,0.95$ and 0.27 , respectively. While at fault clearing, $r_{i a}$, $r_{i b}, r_{i c}$ have values of $-0.57,0.95$ and 0.38 , respectively. The cross-correlation coefficient $r_{i b c}$, calculated between the two faulted phases ( $A$ and $C$ ), at fault start has a value of -1 , whereas its value is equal to -0.5 before fault inception. The value of cross-correlation coefficient, at fault inception, confirms that the fault type is phase-tophase fault. Consequently, our technique can determine the fault type whether double phase-to-ground or phase-tophase fault by calculating the cross-correlation between the two faulted phases. If $r_{a c} \approx-1$, at fault starts, the fault type is phase-to-phase otherwise it is double phase-to-ground. Summary of the correlation coefficients for the measured signals at different periods is shown in Table 4. These results show that the correlation coefficient value at fault initiation is good detector to determine the faulted phases and distinguish between phase-phase isolated and grounded faulty without adding any extra measuring equipments.

Table 4. Correlation coefficients in case of double phase fault $\left(\delta_{l}=20\right.$ degree, double phase fault (b-c-g) with $R_{f}=0$ ohm).

\begin{tabular}{|c|c|c|c|c|c|c|}
\hline Fault type & Signals & (r) pre-fault & (r) at fault start & (r) during fault & (r) at fault clearing & (r) post-fault \\
\hline \multirow{4}{*}{ Double line fault } & ia & ria $=1$ & -0.55 & 1 & -0.57 & 1 \\
\hline & $\mathrm{ib}$ & $\mathrm{rib}=1$ & 0.95 & 1 & 0.95 & 1 \\
\hline & ic & ric $=1$ & 0.27 & 1 & 0.38 & 1 \\
\hline & $\mathrm{ib} \& \mathrm{ic}$ & ribc $=-0.5$ & -1 & -1 & -0.62 & -0.5 \\
\hline
\end{tabular}




\subsection{Three Phase-to-Ground Fault with High Resistance (Case 5)}

This case studies the effect of three-phase-to-ground fault with high fault resistance on the proposed technique. In this case, the applied fault resistance $\left(R_{f}\right)$ is $200 \mathrm{ohm}$. The operating power angle of generator $1\left(\delta_{l}\right)$ is 20 degree. Figure 6 shows the simulation results for case 5 . Figure 6 (a-c) present the instantaneous values for the three-phase currents. In this case, it is noticed that the three-phase currents during the fault are higher than the pre-fault currents; their values are nearly four times the pre-fault currents (these values are less than that are in case "1"). The auto-correlation coefficients $\left(r_{x}\right)$ are calculated between two successive half-cycle for the three-phase current signals. The three-phase current correlation coefficients $r_{i a}, r_{i b}, r_{i c}$ are shown in Fig. 6 (d-f). The values of $r_{i a}, r_{i b}, r_{i c}$ are equal and close to one before fault inception and after fault clearing. At fault start they are equal and less than 0.65 while they are less than 0.74 at fault clearing. From the above results, it is clear that the auto-correlation coefficient values at fault initiation are good detector to determine the faulted phases with high resistance. Also, the correlation coefficient values at fault initiation are higher than that are in case "1".The correlation coefficient values are closely to one for current signals of healthy phase and they are less than one for faulty phase. Summary of the correlation coefficients for the measured current signals at the different periods are shown in Table 5.

Table 5. Correlation coefficients in case of three phase-to-ground fault ( $\delta_{l}=20$ degree, three-phase to ground faults (a-b-c-g) with $\left.R_{f}=200 \mathrm{ohm}\right)$.

\begin{tabular}{lllllll}
\hline Fault type & Signal & (r) pre-fault & (r) at fault start & (r) during fault & (r) at fault clearing & (r) post-fault \\
\hline Three line-to- & ia & ria $=1$ & 0.58 & 1 & 0.65 & 1 \\
ground fault with & ib & rib $=1$ & 0.65 & 1 & 0.47 & 1 \\
high resistance & ic & ric $=1$ & 0.54 & 1 & 0.74 & 1 \\
\hline
\end{tabular}

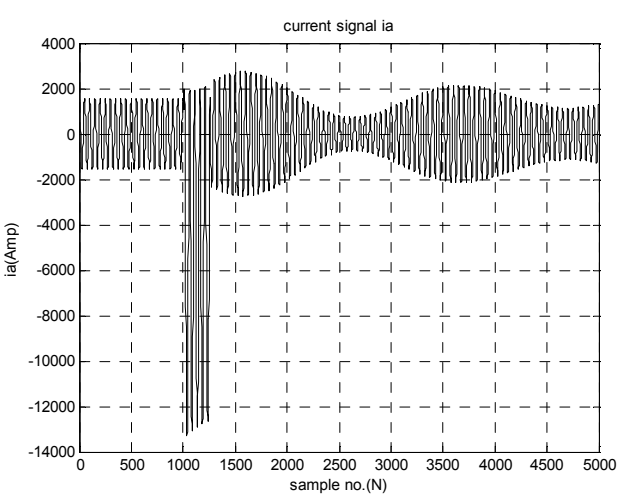

(a) The current ia for case 1 .

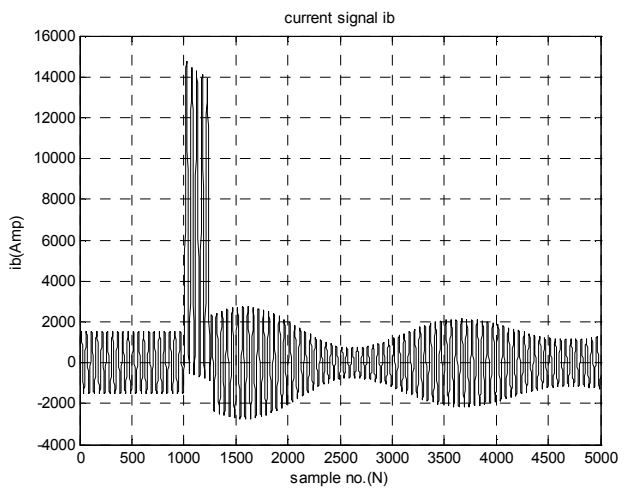

(b) The current ib for case 1 .

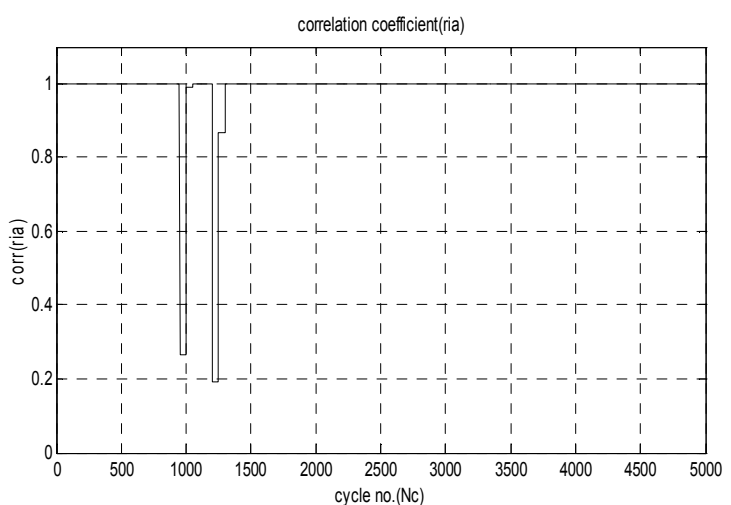

(d) ria for the current ia for case 1.

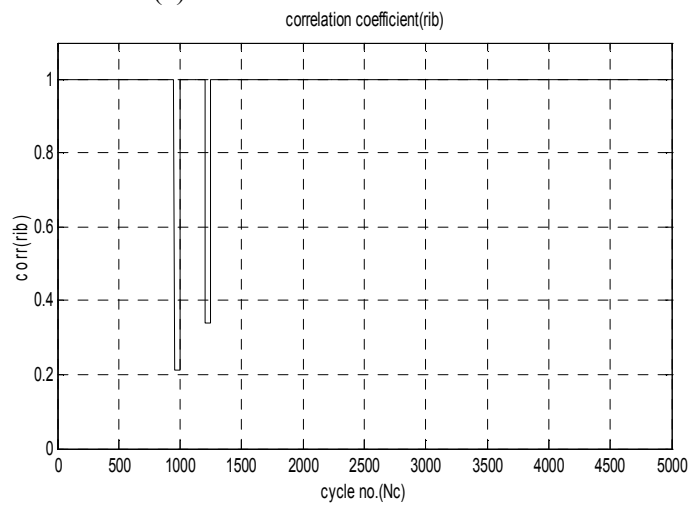

(e) rib for the current ib for case 1. 


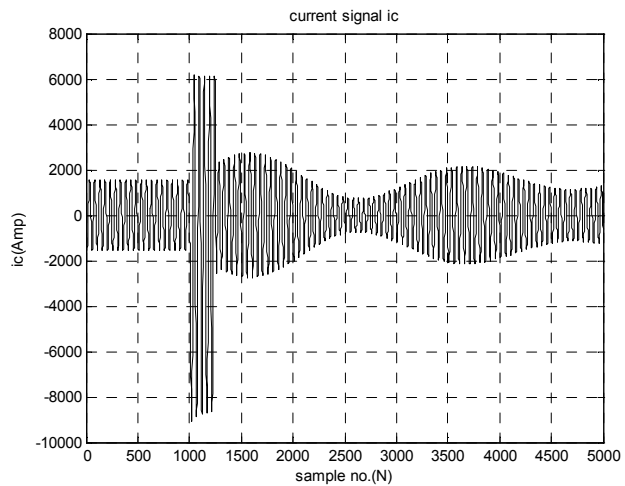

(c) The current ic for case 1 .

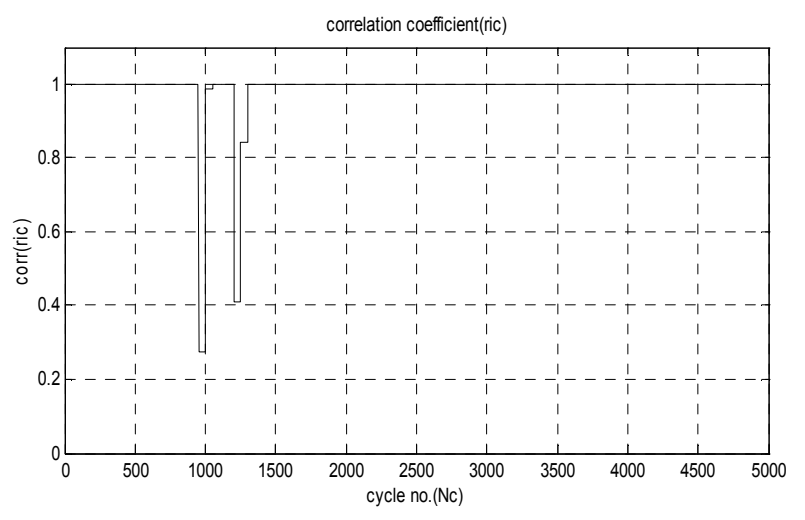

(f) ric for the current ic for case 1.

Figures 2(a-f). The simulation results for case 1, $\delta_{1}=20$ degree, Three-phase to ground faults (a-b-c-g)

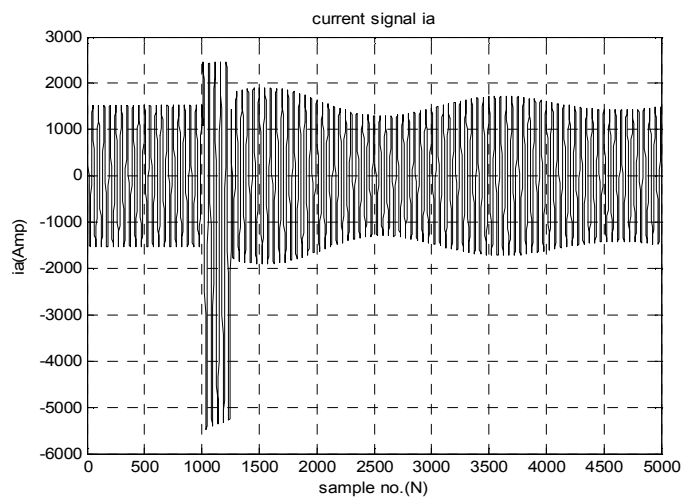

(a) The current ia for case 2 .

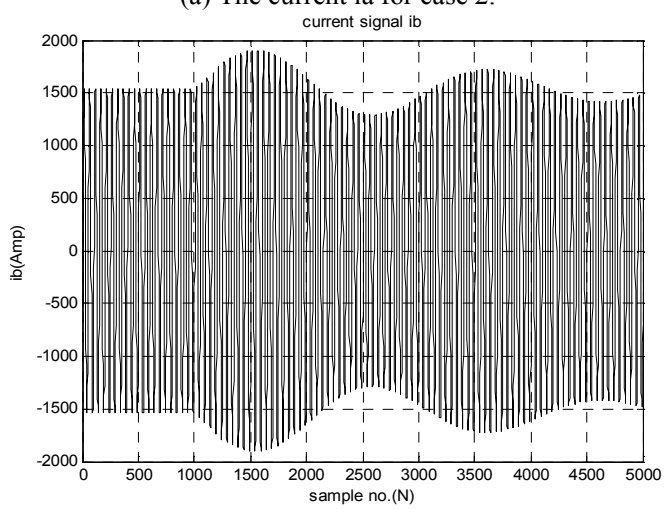

(b) The current ib for case 2 .

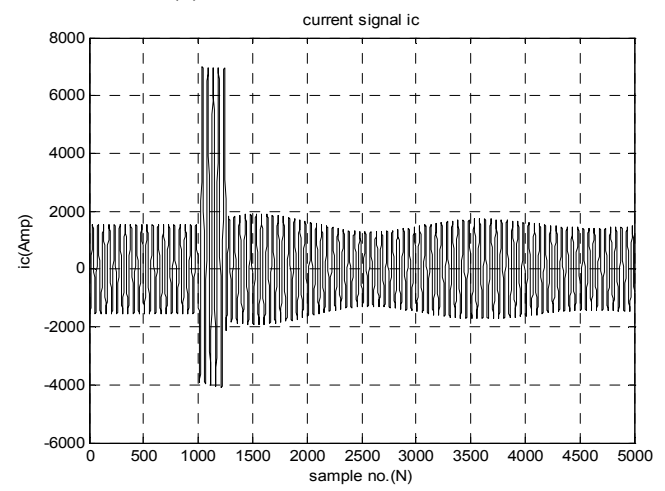

(c) The current ic for case 2

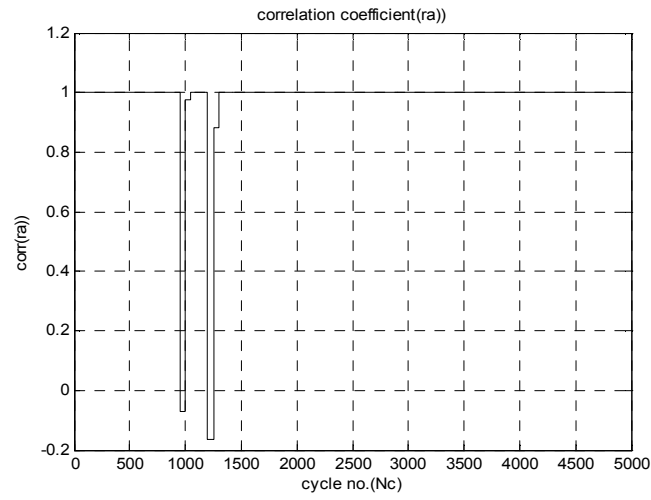

(d) ria for the current ia for case 2 .

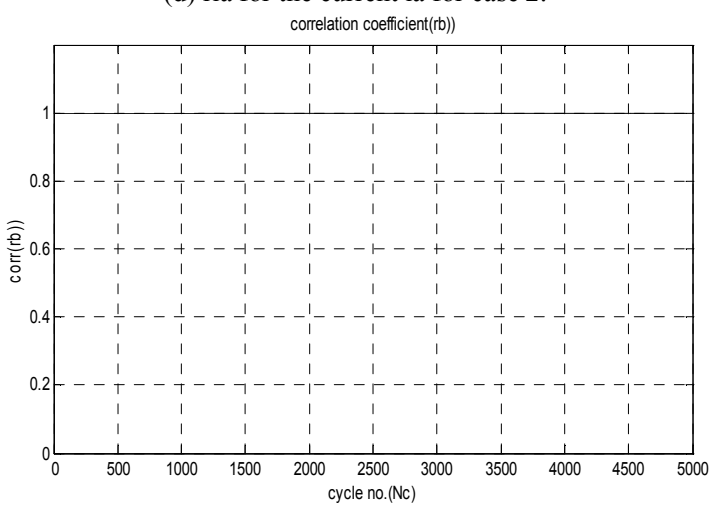

(e) rib for the current ib for case 2 .

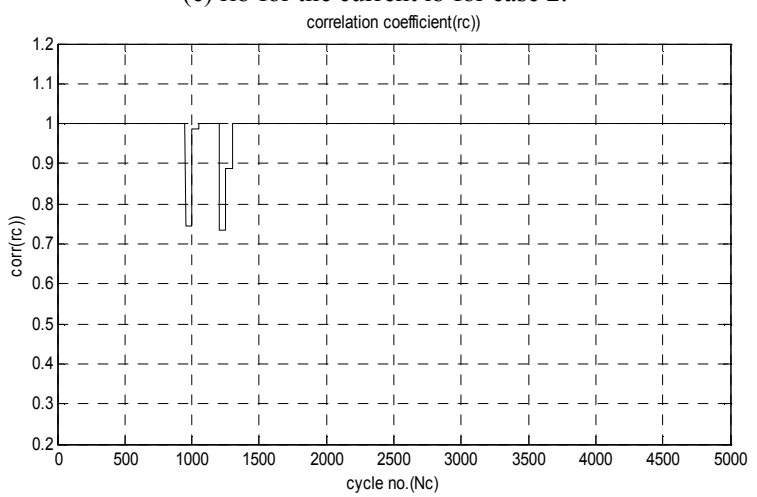

(f) ric for the current ic for case 2.

Figure 3(a-f). The simulation results for case 2, $\delta_{l}=20$ degree, single line-to-ground fault (a-g). 


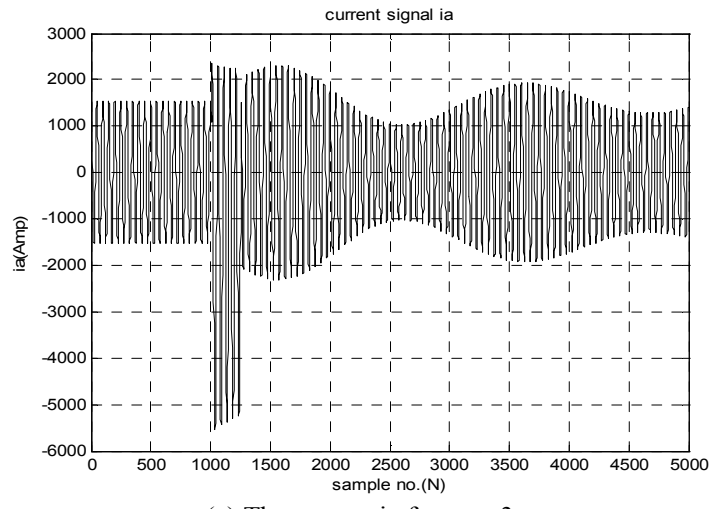

(a) The current ia for case 3 .

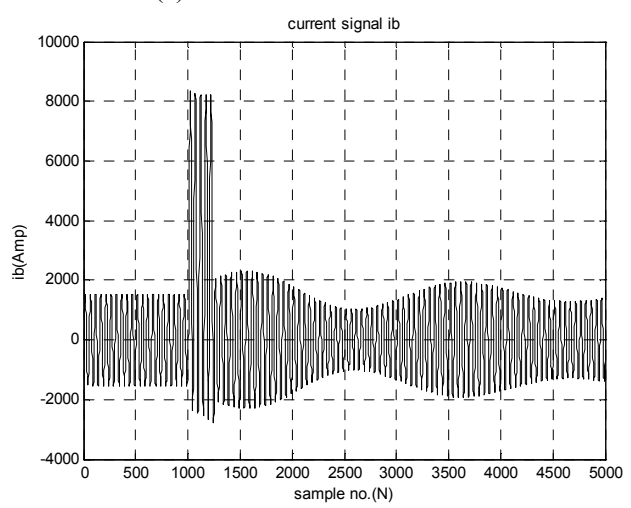

(b) The current ib for case 3 .

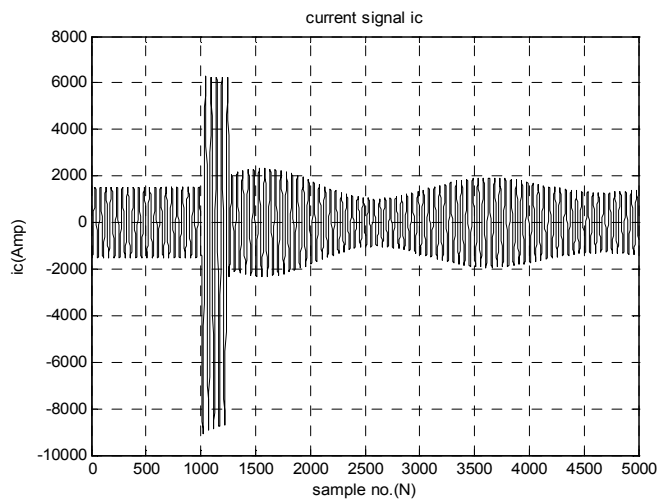

(c) The current ic for case 3 .

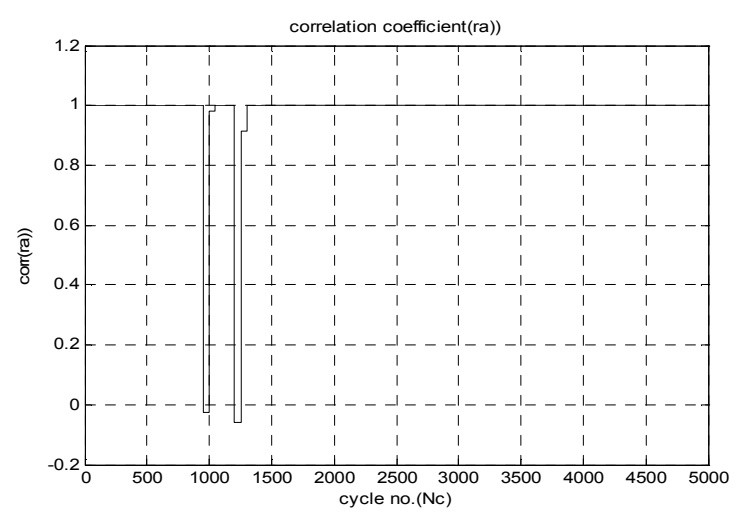

(d) ria for the current ia for case 3 .

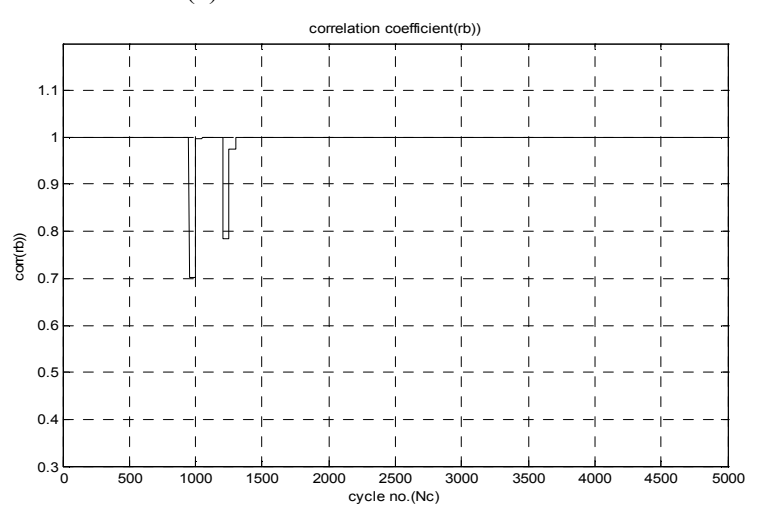

(e) rib for the current ib for case 3.

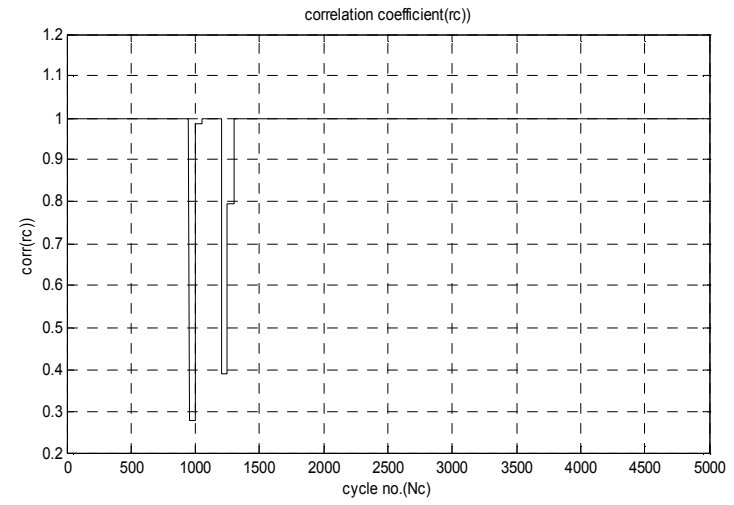

(f) ric for the current ic for case 3 .

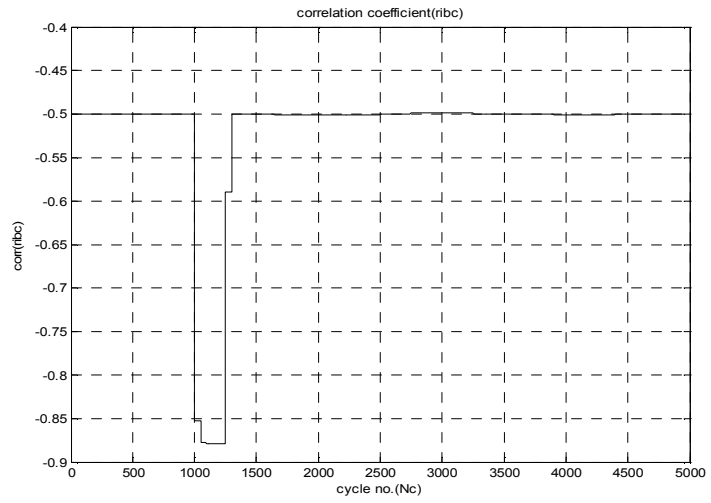

(g) riac for the currents ia \& ic for case 3.

Figures $4(a-g)$. The simulation results for case 3, $\delta_{1}=20$ degree, double line-to-ground fault (a-c-g). 


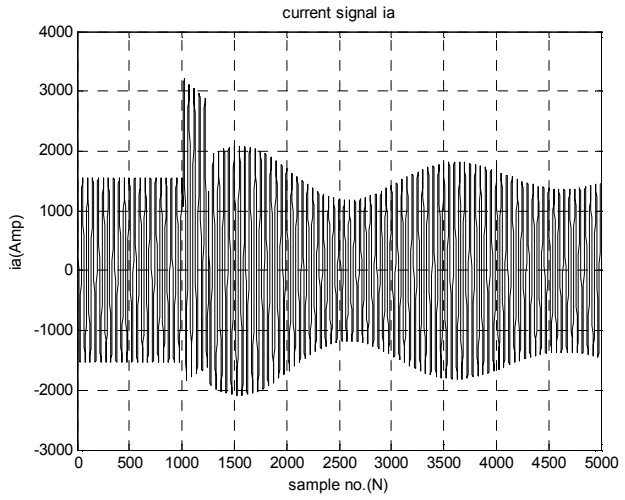

(a) The current ia for case 4.

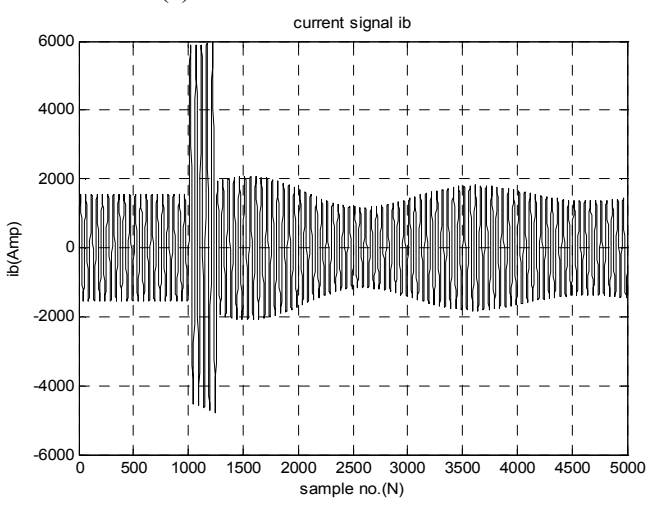

(b) The current ib for case 4 .

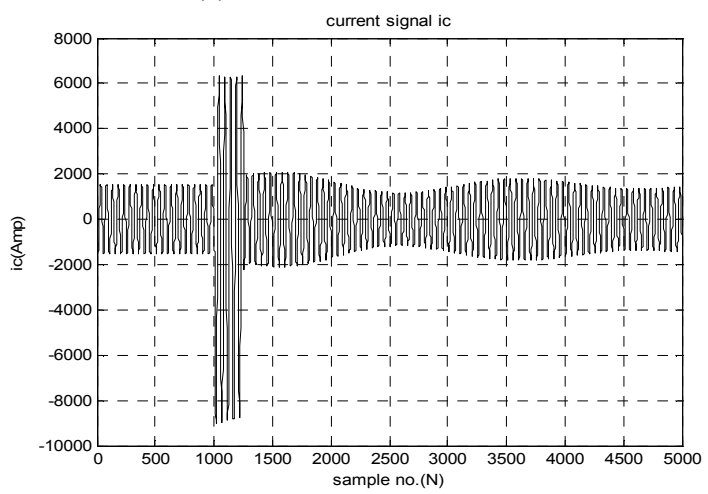

(c) The current ic for case 4 .

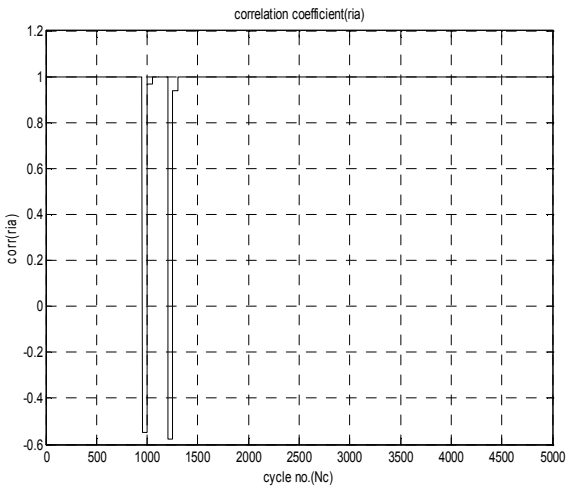

(d) ria for the current ia for case 4.

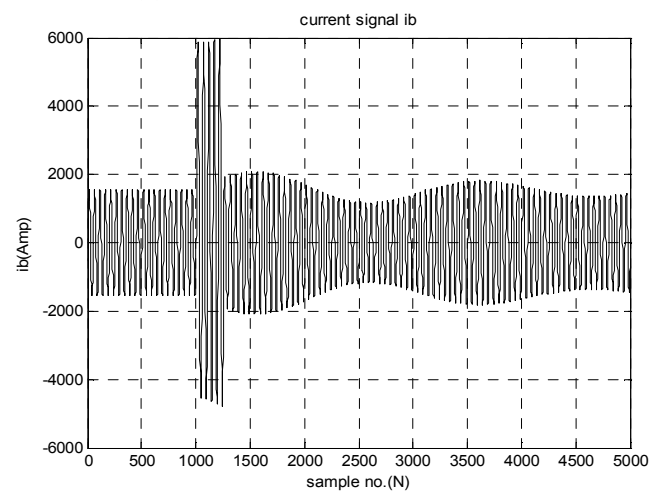

(e) rib for the current ib for case 4 .

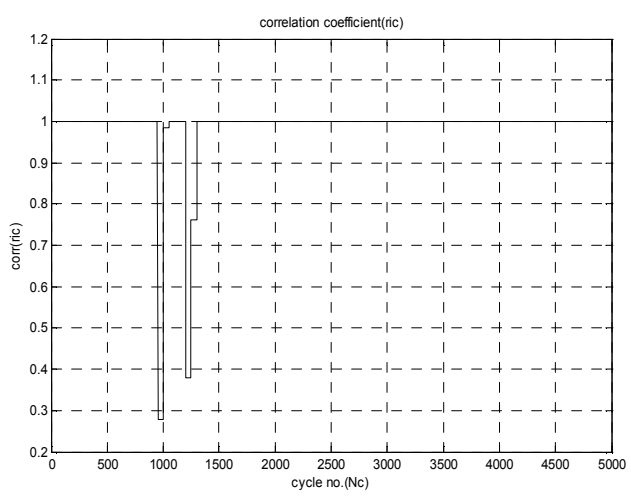

(f) ric for the current ic for case 4.

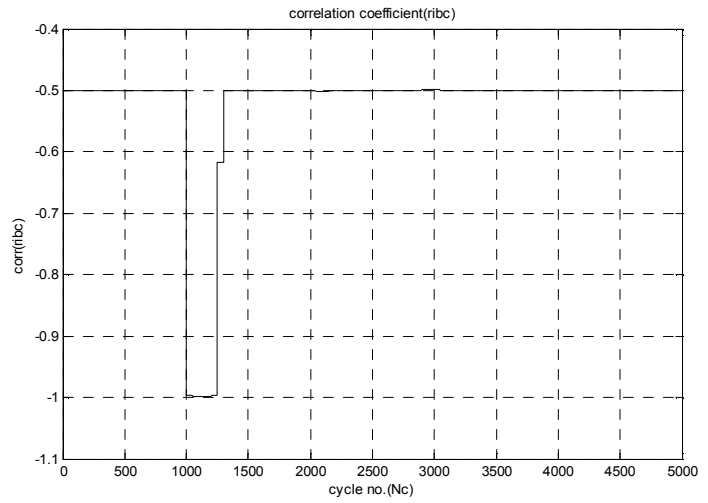

(g) riac for the currents ia $\&$ ic for case 4.

Figures 5(a-g). The simulation results for case 4, $\delta_{1}=20$ degree, double line fault (a-c). 


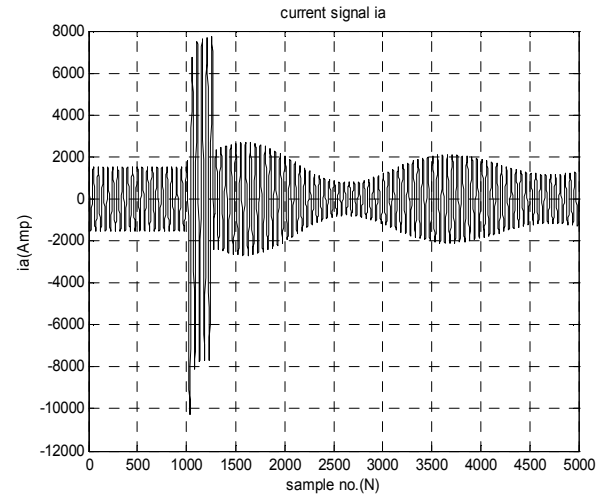

(a) The current ia for case 5

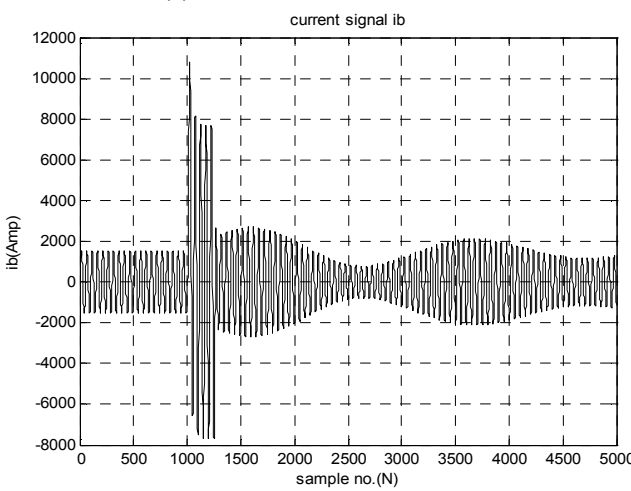

(b) The current ib for case 5

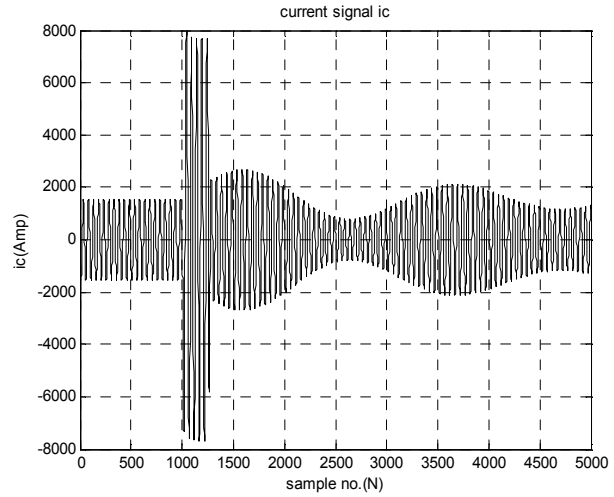

(c) The current ic for case 5

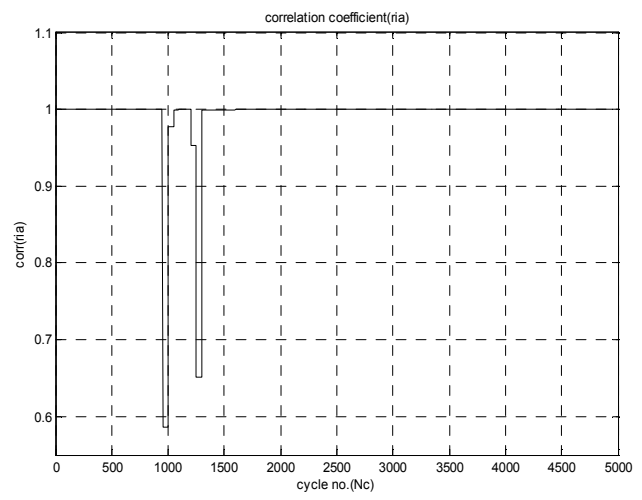

(d) ria for the current ia for case 5

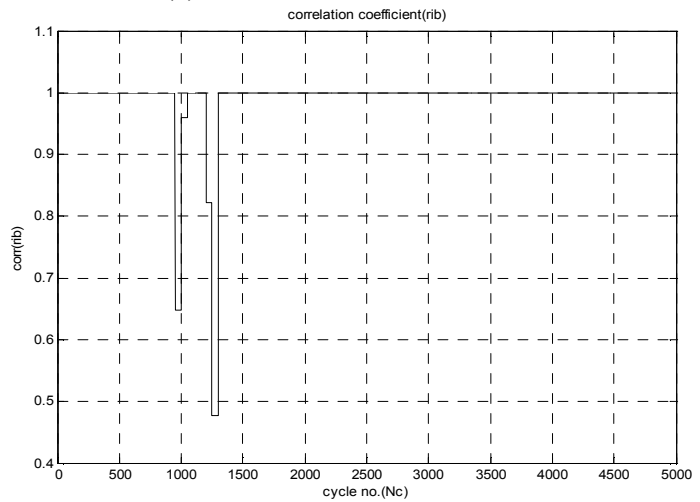

(e) rib for the current ib for case 5

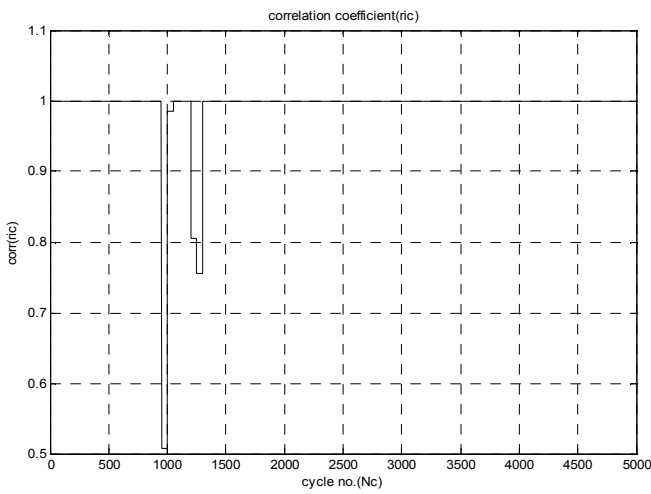

(f) ric for the current ic for case 5

Figures 6(a-f). The simulation results for case 5, $\delta_{l}=20$ degree, Three-phase to ground faults (a-b-c-g) with fault resistance $\left(R_{f}\right)=200 \mathrm{ohm}$.

From the comparison between case "1" and case "5" we deduce the following findings:

(a) The greater the applied fault resistance $\left(R_{f}\right)$ the lower the DC components in the fault current (because of the lower primary time constant of the power system, $\left(\tau_{p}\right.$ $\left.=X_{L} / \omega R\right)$.

(b) The greater the applied fault resistance $\left(R_{f}\right)$ the greater the correlation coefficient values at fault initiation.

(c) The greater the applied fault resistance $\left(R_{f}\right)$ the greater the degree of power system stability.

\section{Simulation Results and Technique Evaluation}

From the different case studies, we summarize the following:
(1) Faults cause transient of the transmission line's currents and as a result cause collapse for power system voltage magnitude.

(2) The proposed technique is based on the two types of algorithms for fault detection:

Algorithm 1: Fault detection using superimposed quantities (delta algorithm)

Algorithm 2: Fault detection using auto-correlation coefficient value

(3) Three tasks are starting in parallel for fault detection, fault classifications and faulty phase selection.

(4) Auto-correlation coefficient between two successive half-cycles with the same polarity for each phase current signal can be used to identify the faulted phase status. The cross-correlation coefficient between the two faulted 
phases is useful for determining the type of fault either double phase-to-ground or phase-to-phase fault.

(5) An extensive simulation studies are done to study the effects of different loading levels, fault resistances $\left(R_{f}\right)$ and fault inception angle on the performance of the proposed technique. The results show that our technique has high accuracy and efficiency for fault detection and classification with a wide range of fault resistances from zero value up to $500 \Omega$.

\section{Conclusions}

In this paper, a correlation technique of transmission line for fault identification and faulty phase selection has been proposed. The main achievements of this work are as follows:

1. Three line current measurements are sufficient to implement this technique.

2. It is accurate to identify all ten types of short-circuit faults.

3. It is efficient to distinguish between phase-phase isolated and grounded faulty without needing any extra equipment.

4. The reliability of the proposed method is quite high.

5. It is quite effective over a wide range of a pre-fault power level, fault resistance, and fault inception angle.

6. Fast and simple method, as the time taken by this method is about $10 \mathrm{~ms}$ (for a $50-\mathrm{Hz}$ power system).

7. The effects of DC components and harmonics are eliminated with estimation of correlation coefficients.

8. The faulted phases can be determined by using correlation technique.

9. The technique does not use the data of power system element but it needs only three phase current measurements available at the relay location.

\section{References}

[1] M. M. Saha et al., "A new accurate fault location algorithm for series compensated lines," IEEE Trans. Power Delivery, vol. 14, pp. 789-797, July 1999.

[2] A. G. Phadke, Computer Relaying for Power Systems. New York: Wiley, 1988.

[3] R. K. Aggrawal, Q. Y. Xuan, R. W. Dunn, and A. Bennett, "A novel fault classification technique for double-circuit line based on a combined unsupervised/supervised neural network," IEEE Trans. Power Delivery, vol. 14, pp. 1250 125, Oct. 1999.

[4] W.-M. Lin, C.-D. Yang, and J. H. Lin, "A fault classification method by RBF neural network with OLS learning procedure," IEEE Trans. Power Delivery, vol. 16, pp. 473477, Oct. 2001.
[5] T. Dalstein and B. Kulicke, "Neural network approach to fault classification for high speed protective relaying," IEEE Trans. Power Delivery, vol. 10, pp. 1002-1011, Apr. 1995.

[6] D. K. Ranaweera, "Comparison of neural network models for fault diagnosis of power system," Elect. Power Syst. Res., pp. 99-104, 1994.

[7] K. H. Kim and J. K. Park, "Application of hierarchical neural networks to fault diagnosis of power system," Int. J. Elect. Power Energy Syst., vol. 15, no. 2, pp. 65-70, 1993.

[8] A. L. O. Fernandez and N. K. I. Ghonaim, "A novel approach using a FIRANN for fault detection and direction estimation for high voltage transmission lines," IEEE Trans. Power Delivery, vol. 17, pp. 894-901, Oct. 2002.

[9] A. Poeltl and K. Frohich, "Two new methods for fast fault type detection by means of parameters fitting and artificial neural networks," IEEE Trans. Power Delivery, vol. 14, pp. 1269-1275, Oct. 1999.

[10] A. A. Girgis and M. B. Johns, "Ahybrid expert system for faulted section identification, fault type classification and selection of fault location algorithms," IEEE Trans. Power Delivery, vol. 4, pp. 978-985, Apr. 1989.

[11] C. A. Protopapas, K. P. Psatiras, and A. V. Machias, "An expert system for substation fault diagnosis and alarm processing," IEEE Trans. Power Delivery, vol. 6, pp. 648655, Apr. 1991.

[12] H. T. Yang, W. Y. Chang, and C. L. Huang, "On line fault diagnosis of power substation using connectionist expert system," IEEE Trans. Power Syst., vol. 10, pp. 323-331, Feb. 1995.

[13] A. Ferrero, S. Sangiovanni, and E. Zapitelli, "A fuzzy set approach to fault type identification in digital relaying," IEEE Trans. Power Delivery, vol. 10, pp. 169-175, Jan. 1995.

[14] H.Wang andW.W. L. Keerthipala, "Fuzzy neuro approach to fault classification for transmission line protection," IEEE Trans. Power Delivery, vol. 13, pp. 1093-1104, Oct. 1998.

[15] T. Adu, "An accurate fault classification technique for power system monitoring devices," IEEE Trans. Power Delivery, vol. 17, pp. 684-690, July 2002.

[16] M.E. Masoud, M.M.A. Mahfouz, "Protection scheme for transmission lines based on alienation coefficients for current signals", IET Gener. Transm. Distrib., Vol. 4, Iss. 11, pp. 1236 - 1244. March 2010.

[17] W. Hauschild, and W. Mosch, "Statistical Techniques for High Voltage Engineering", hand book, English edition published by peter pere grinus Ltd., London, United Kingdom, chapter 2, pp. 78-79, 1992.

[18] Instruction Manual for Generator Electrical Equipment, Upper Egypt Electricity Production Company, Elkureimat $\Pi$ 750 MW Combined Cycle Project, Steam Turbine Generator \& Auxiliaries (Generator Electrical Equipment), Hitachi, Ltd., Tokyo Jaban.

[19] ATP - version 3.5 for Windows 9x/NT/2000/XP - Users' Manual-Preliminary Release No. 1.1 - October 2002. 